Research Article

\title{
Bifurcation of Traveling Wave Solutions of the Dual Ito Equation
}

\author{
Xinghua Fan and Shasha Li \\ Faculty of Science, Jiangsu University, Zhenjiang, Jiangsu 212013, China \\ Correspondence should be addressed to Xinghua Fan; fan131@vip.sina.com
}

Received 9 May 2014; Accepted 15 July 2014; Published 5 August 2014

Academic Editor: Yonghui Xia

Copyright (C) 2014 X. Fan and S. Li. This is an open access article distributed under the Creative Commons Attribution License, which permits unrestricted use, distribution, and reproduction in any medium, provided the original work is properly cited.

The dual Ito equation can be seen as a two-component generalization of the well-known Camassa-Holm equation. By using the theory of planar dynamical system, we study the existence of its traveling wave solutions. We find that the dual Ito equation has smooth solitary wave solutions, smooth periodic wave solutions, and periodic cusp solutions. Parameter conditions are given to guarantee the existence.

\section{Introduction}

Study on two-component equations has drawn a lot of interest among researchers [1-6]. The two-component equation we are going to discuss in the present paper is the dual Ito equation [7]

$$
\begin{gathered}
u_{t} \pm u_{x x t}=3 u u_{x}+v v_{x}+\left(u u_{x x}+\frac{1}{2} u_{x}^{2}\right)_{x} \\
v_{t}=(u v)_{x} .
\end{gathered}
$$

The dual Ito equation (1) is of interest due to the connection of the Ito equation with the KdV equation.

The Ito equation [8],

$$
\begin{gathered}
u_{t}-u_{x x x}=3 u u_{x}+v v_{x} \\
v_{t}=(u v)_{x},
\end{gathered}
$$

is a prototypical example of a two-component $\mathrm{KdV}$ equation. It is shown that the coupled equation possesses infinitely many symmetries and conservation laws. It is also shown that these symmetries define a hierarchy of the coupled equation each of which is a Hamiltonian system.

By the sense of "tri-Hamiltonian duality" [7], the celebrated Camassa-Holm equation $[9,10]$

$$
u_{t} \pm u_{x x t}=3 u u_{x}+\left(u u_{x x}+\frac{1}{2} u_{x}^{2}\right)_{x}
$$

can be seen as the dual equation of the KdV equation

$$
u_{t}=u_{x x x}+3 u u_{x} .
$$

Taking plus sign in (3) leads to an integrable equation which supports compactons, whereas minus sign is the water wave model derived by Camassa-Holm, whose bounded travelling waves (termed peakons) develop a discontinuity in the first derivatives [10].

Different methods have been used to study exact solutions to standard Ito equation or generalized ones as well as higherorder Ito equation [11-17]. But there are fewer researches about traveling wave solutions of (1). We want to know, for (1), whether there exist some interesting solutions such as smooth soliton, peakon, cuspon, and compacton [18] solutions.

In this paper, we will apply the method of dynamical system [19-24] from a mathematical point of view to study the traveling wave solutions of (1).

The rest of this paper is organized as follows. In Section 2, after transforming the two-component dual Ito equation into a planar dynamical system, we discuss the bifurcation conditions and possible phase portraits of the planar system. Based on those phase portraits, Section 3 presents different types of solutions such as smooth solitary wave solutions, smooth periodic wave solutions, and periodic cusp solutions. The last section is devoted to a short conclusion. 


\section{Bifurcation Conditions and Possible Phase Portraits}

In this section, the properties of equilibrium points and possible phase portraits will be given.

We consider the traveling wave solutions of (1) in the form

$$
u(x, t)=\phi(\xi), \quad v(x, t)=\psi(\xi), \quad \xi=x-c t,
$$

where $c$ is the wave speed.

Substituting (5) into (1), we get a system of ordinary differential equations

$$
\begin{gathered}
c \phi^{\prime} \pm c \phi^{\prime \prime \prime}+3 \phi \phi^{\prime}+\psi \psi^{\prime}+2 \phi^{\prime} \phi^{\prime \prime}+\phi \phi^{\prime \prime \prime}=0, \\
c \psi^{\prime}+\psi \phi^{\prime}+\phi \psi^{\prime}=0
\end{gathered}
$$

where "' " is the derivative with respect to $\xi$.

Integrating (6) once with respect to $\xi$, we obtain

$$
\begin{gathered}
c \phi+\frac{3}{2} \phi^{2}+\frac{1}{2} \phi^{\prime 2}+(c+\phi) \phi^{\prime \prime}+\frac{1}{2} \psi^{2}+g=0, \\
\phi \psi+c \psi+r=0,
\end{gathered}
$$

where $g, r$ are integration constants.

From the second equation of (7), we get

$$
\psi=-\frac{r}{c+\phi(\xi)} .
$$

Plugging (8) into the first equation of (7), we obtain a nonlinear ODE

$$
\begin{aligned}
\left(2 \phi^{3}\right. & \left.+a c \phi^{2}+a c^{2} \phi+2 c^{3}\right) \phi^{\prime \prime}+(\phi+c)^{2}\left(\phi^{\prime}\right)^{2}+3 \phi^{4}+8 c \phi^{3} \\
& +\left(7 c^{2}+2 g\right) \phi^{2}+\left(4 c g+2 c^{3}\right) \phi+r^{2}+2 g c^{2}=0,
\end{aligned}
$$

where $a=6$ when (1) takes plus sign and $a=2$, minus sign.

Letting $y=\phi^{\prime}$, we get the following planar system:

$$
\begin{aligned}
\frac{d \phi}{d \xi}= & y \\
\frac{d y}{d \xi}= & \left(\left(3 \phi^{4}+8 c \phi^{3}+\left(y^{2}+7 c^{2}+2 g\right) \phi^{2}\right.\right. \\
& \left.\quad+2 c\left(y^{2}+c^{2}+2 g\right) \phi+y^{2} c^{2}+2 g c^{2}+r^{2}\right) \\
& \left.\times\left(2(c \pm \phi)(\phi+c)^{2}\right)^{-1}\right) .
\end{aligned}
$$

System (10) is a planar dynamical system defined in a 3 -parameter space $(c, g$, and $r)$. Because the phase orbits defined by the vector fields of system (10) determine all traveling wave solutions, we will investigate the bifurcations of phase portraits of these systems in the phase plane $(\phi, y)$ as the parameters are changed.

System (10) has a singular straight line $\phi=-c$ when taking plus sign or two singular straight lines $\phi= \pm c$ when taking minus sign. To avoid the singularity, letting $d \xi=2(c \pm$ $\phi)(c+\phi)^{2} d \tau$, system (10) is changed to a regular system:

$$
\begin{aligned}
\frac{d \phi}{d \tau}=2(c \pm \phi)(c+\phi)^{2} y \\
\frac{d y}{d \tau}=\mp\left((\phi+c)^{2} y^{2}+3 \phi^{4}+8 c \phi^{3}+\left(7 c^{2}+2 g\right) \phi^{2}\right. \\
\left.+\left(4 c g+2 c^{3}\right) \phi+r^{2}+2 g c^{2}\right) .
\end{aligned}
$$

Now we consider the equilibrium points of system (10) lying on the $\phi$-axis. Let

$$
\begin{aligned}
F(\phi)= & 3 \phi^{4}+8 c \phi^{3}+\left(2 g+7 c^{2}\right) \phi^{2} \\
& +\left(4 g c+2 c^{3}\right) \phi+2 g c^{2}+r^{2}, \\
G(\phi)= & \phi^{4}+2 c \phi^{3}+\left(2 g+c^{2}\right) \phi^{2}+2 g c \phi-r^{2} .
\end{aligned}
$$

We see that $F(\phi)$ has at most four real roots $\phi_{i}, i=1, \ldots, 4$, since it is a polynomial with order four. Then system (10) has at most four equilibrium points $E_{i}\left(\phi_{i}, 0\right)$.

We need to find the bifurcation conditions for the parameters. Equation $F^{\prime}(\phi)=0$ has three roots $-c, \phi_{ \pm}=$ $-c / 2 \pm \sqrt{3 c^{2}-12 g} / 6$, if $g<(1 / 4) c^{2}$. It means we only need to discuss the situation when $g<(1 / 4) c^{2}$. Thus we get a bifurcation condition $g=(1 / 4) c^{2}$. When $g=-(1 / 2) c^{2}$, we have $\phi_{-}=-c$. Therefore $g=-(1 / 2) c^{2}$ is another bifurcation condition.

Next we will study possible order of those roots. We see that $F(-c)=r^{2}>0$, and $F\left(\phi_{+}\right)-F\left(\phi_{-}\right)=(c / 9)(4 g-$ $\left.c^{2}\right) \sqrt{3 c^{2}-12 g}<0$. If $F\left(\phi_{+}\right) \leq 0$ and $g<(1 / 4) c^{2}$, then $F^{\prime \prime}(-c)=2 c^{2}+4 g, F^{\prime \prime}\left(\phi_{+}\right)=2 c^{2}+2 c \sqrt{3 c^{2}-12 g}-8 g$, and $F^{\prime \prime}\left(\phi_{-}\right)=2 c^{2}-2 c \sqrt{3 c^{2}-12 g}-8 g$. When $-(1 / 2) c^{2} \leq g<$ $(1 / 4) c^{2}$, we have $-c \leq \phi_{-}$; then $-c<\phi_{-}<\phi_{1}<\phi_{+}<\phi_{2}$. Furthermore, if $F\left(\phi_{+}\right)=0, \phi_{1}$ meets $\phi_{+}$. If $F\left(\phi_{+}\right)>0$, there is no zeros for $F(\phi)$. If $g>(1 / 4) c^{2}$, there is only one zero $-c$ for $F^{\prime}$, and there is no equilibrium point. If $g=(1 / 4) c^{2}$, then $\phi_{+}=\phi_{-}$. There are two zeros $-c$ and $-(1 / 2) c$ for $F^{\prime}$. Because $F^{\prime \prime}\left(\phi_{ \pm}\right)=0, F^{\prime \prime \prime}\left(\phi_{ \pm}\right)=12 c$, there are zeros for $F(\phi)$.

Now consider singular equilibrium points on the singular lines. On the singular line $\phi=-c$ there are no singular equilibria if $r \neq 0$. When (1) takes the minus sign and $F(c)=$ $20 c^{4}+8 g c^{2}+r^{2}<0$, on the singular line $\phi=c$, there are two singular equilibria $E_{5,6}=\left(c, \pm y_{c}\right)$ where $y_{c}=\sqrt{-F(c)} / 2 c$.

To investigate the equilibrium points of (11), let $M\left(\phi_{e}, y_{e}\right)$ be the coefficient matrix of the linearized system of system (11) at the equilibrium point $\left(\phi_{e}, y_{e}\right) ; J\left(\phi_{e}, y_{e}\right)=$ det $M\left(\phi_{e}, y_{e}\right)$ is the determinant. We have

$$
J(\phi, y)=-2(c+\phi)^{3}\left(4 y^{2} c+4 y^{2} \phi-\frac{d}{d \phi} F(\phi)\right)
$$


corresponding to plus sign in (1) and

$$
\begin{aligned}
J(\phi, y)= & -2(\phi+c)^{2} \\
& \times\left(4 y^{2} c \phi+c \frac{d}{d \phi} F(\phi)+4 y^{2} \phi^{2}-\phi \frac{d}{d \phi} F(\phi)\right)
\end{aligned}
$$

corresponding to minus sign in (1). We can get $J(\phi, 0)=2(\phi \pm$ $c)(c+\phi)^{2}(d / d \phi) F(\phi)$ and $p=\operatorname{trace}(J)=4(c \pm \phi)(c+\phi) y$, respectively.

By the theory of planar dynamical systems [22], we know that an equilibrium point of a planar Hamiltonian system is a saddle point in the case of $J<0$, a center in the case of $J>0$, and a cusp in the case of $J=0$, and its Poincare index is 0 .

2.1. Equilibrium Points and Phase Portraits of (10) When Taking Plus Sign in (1). Except for the straight line $\phi=-c$ or $\phi= \pm c$, systems (10) and (11) have the same first integral

$$
\begin{aligned}
H(\phi, y) & =\frac{b y^{2} \pm\left(\phi^{4}+2 c \phi^{3}+\left(c^{2}+2 g\right) \phi^{2}+2 g c \phi-r^{2}\right)}{c+\phi} \\
& =h,
\end{aligned}
$$

where $b=(\phi+c)^{2}$ corresponding to plus sign in (1) and $b=$ $c^{2}-\phi^{2}$ corresponding to minus sign.

Let $h_{i}=H\left(\phi_{i}, 0\right), h_{s}=H\left(c, \pm y_{c}\right)$. We can get $h_{s}=$ $-G(c) / 2 c$, where $G(c)=4 c^{4}+4 g c^{2}-r^{2}$.

System (11) has the same phase portraits as system (10) except for $\phi=-c$ corresponding to plus sign or $\phi= \pm c$ corresponding to minus sign.

Lemma 1. When $F\left(\phi_{+}\right)<0$ and $-(1 / 2) c^{2} \leq g<(1 / 4) c^{2}$, there are two equilibrium points $E_{1}\left(\phi_{1}, 0\right)$ and $E_{2}\left(\phi_{2}, 0\right)\left(\phi_{1}<\right.$ $\left.\phi_{2}\right) . E_{1}$ is a saddle point and $E_{2}$ is a center point. In this parameter condition, a branch of the level curve $H(\phi, y)=h_{1}$ defines a homoclinic orbit to the saddle point $E_{1}$, and a branch of the level curve $H(\phi, y)=h$ with $h \in\left(h_{2}, h_{1}\right)$ gives rise to a family of smooth periodic orbits of (10) (see Figure 1(a)).

Lemma 2. When $g<-(1 / 2) c^{2}$, the equilibrium points of system (10) can be described by the following cases.

(1) If $F\left(\phi_{-}\right)<0$ and $F\left(\phi_{+}\right)<0$, there are four equilibrium points $E_{i}\left(\phi_{i}, 0\right), i=1, \ldots, 4,\left(\phi_{1}<\phi_{-}<\phi_{2}<-c<\right.$ $\left.\phi_{3}<\phi_{+}<\phi_{4}\right) . E_{1}$ and $E_{4}$ are centers and $E_{2}$ and $E_{3}$ are saddle points. There is a homoclinic orbit defined by $H(\phi, y)=h_{i}, i=2,3$, passing through $E_{2}$ and $E_{3}$, respectively. There is a family of smooth periodic orbits of (10) enclosing the center $E_{1}$ and $E_{4}$, respectively (see Figure 1(b)).

(2) If $F\left(\phi_{-}\right)>0$ and $F\left(\phi_{+}\right)<0$, there are two equilibrium points $E_{1}\left(\phi_{1}, 0\right)$ and $E_{2}\left(\phi_{2}, 0\right)\left(\phi_{-}<-c<\phi_{1}<\phi_{+}<\right.$ $\left.\phi_{2}\right) . E_{1}$ is a saddle point while $E_{2}$ is a center point. There is a homoclinic orbit defined by $H(\phi, y)=h_{1}$ passing through $E_{1}$. There is a family of smooth periodic orbits of $(10)$ defined by $H(\phi, y)=h, h \in\left(h_{2}, h_{1}\right)$ enclosing the center $E_{2}$ (see Figure 1(c)).

2.2. Equilibrium Points and Phase Portraits of (10) When Taking Minus Sign in (1). When taking minus sign, there are two singular lines of (10). Two more equilibrium points appear. When $F(c)>0$, no equilibrium points exist on the singular line $\phi=c$. If $F\left(\phi_{+}\right)<0$ and $-(1 / 2) c^{2}<g<(1 / 4) c^{2}$, we have $-c<\phi_{-}<\phi_{1}<\phi_{+}<\phi_{2}$. When $g=-(1 / 2) c^{2}$, we have $\phi_{-}=-c<\phi_{1}<\phi_{+}<\phi_{2}$. If $F\left(\phi_{+}\right)=0$, there is only one equilibrium point $E_{1}\left(\phi_{1}, 0\right),\left(-c<\phi_{-}<\phi_{1}=\phi_{+}\right)$which is a saddle piont. If $F\left(\phi_{+}\right)>0$, there are no equilibrium points.

Lemma 3. When $F(c)>0, F\left(\phi_{+}\right)<0$ and $-(1 / 2) c^{2} \leq g<$ $(1 / 4) c^{2}$, system $(10)$ has two equilibrium points $E_{1}\left(\phi_{1}, 0\right)$ and $E_{2}\left(\phi_{2}, 0\right) . E_{1}$ is a center point while $E_{2}$ is a saddle point. There is a homoclinic orbit defined by $H(\phi, y)=h_{2}$ to the saddle $E_{2}$. There is a family of smooth periodic orbits defined by $H(\phi, y=$ $\left.h, h \in\left(h_{1}, h_{2}\right)\right)$ surrounding $E_{2}($ see Figure $2(a))$.

When $F(c)<0$, there is equilibrium at $\phi=c$. When $g<$ $-(1 / 2) c^{2}$, we have $\phi_{+}>0$ and $\phi_{-}<-c<\phi_{+}$; then $f^{\prime \prime}(-c)>0$, where $-c$ is minimum.

Lemma 4. When $F(c)<0$ and $g<-(1 / 2) c^{2}$, the equilibrium points of system (10) can be described by the following cases.

(1) If $F\left(\phi_{-}\right)<0$ and $F\left(\phi_{+}\right)<0$, there are six equilibrium points $E_{i}\left(\phi_{i}, 0\right), i=1, \ldots, 4, E_{5}\left(c, y_{c}\right)$, and $E_{6}\left(c,-y_{c}\right)\left(\phi_{1}<\phi_{-}<\phi_{2}<-c<\phi_{3}<\phi_{+}<\phi_{4}\right)$. $E_{1}, E_{3}$, and $E_{4}$ are centers; $E_{2}, E_{5}$, and $E_{6}$ are saddle points. Equilibrium points $E_{5}$ and $E_{6}$ lie on the singular line $\phi=c$. There is a homoclinic orbit passing through $E_{2}$ enclosing the center $E_{1}$. There is a family of periodic orbits surrounding each center point. There is a singular close orbit passing the singular saddle points $E_{5}$ and $E_{6}($ Figure 2(b)).

(2) If $F\left(\phi_{-}\right)>0$ and $F\left(\phi_{+}\right)<0$, there are four equilibrium points $E_{i}\left(\phi_{1}, 0\right), i=1,2, E_{3}\left(c, y_{c}\right)$, and $E_{4}\left(c,-y_{c}\right)$ $\left(\phi_{-}<-c<\phi_{1}<\phi_{+}<\phi_{2}\right) . E_{1}$ and $E_{2}$ are center points while $E_{3}$ and $E_{4}$ are saddle points on the singular line $\phi=c$. There is a family of smooth periodic orbits surrounding the centers $E_{1}$ and $E_{2}$, respectively. There are two bizarre periodic orbits passing through $E_{3}$ and $E_{4}$ (see Figure 2(c)).

\section{Different Kinds of Traveling Solutions to (1)}

In this section we will give some types of interesting solutions to (1).

Suppose that $u=\phi(\xi)$ is a traveling wave solution for (9) for $\xi \in(-\infty, \infty), \lim _{\xi \rightarrow-\infty} \phi(\xi)=\alpha$, and $\lim _{\xi \rightarrow \infty} \phi(\xi)=\beta$, where $\alpha$ and $\beta$ are two constants. If $\alpha=\beta$, then $\phi(\xi)$ is called a solitary wave solution. Usually, a solitary wave solution for (9) corresponds to a homoclinic orbit of system (10) while a periodic solution for (9) corresponds to a closed orbit of system (10). 


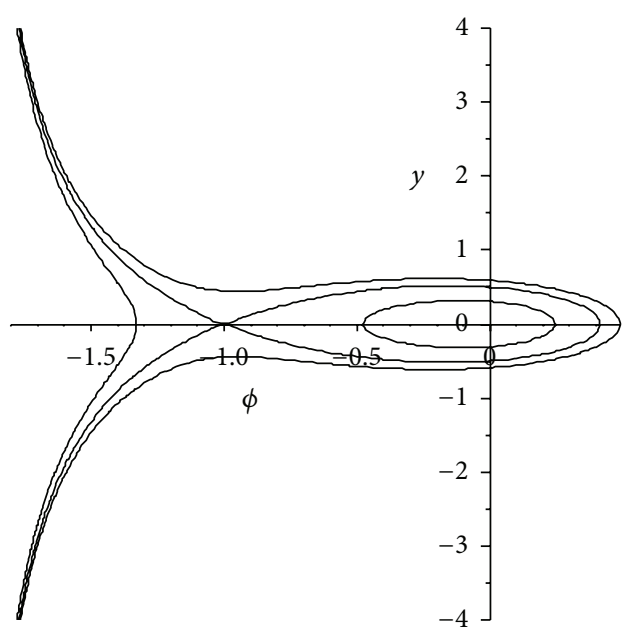

(a)

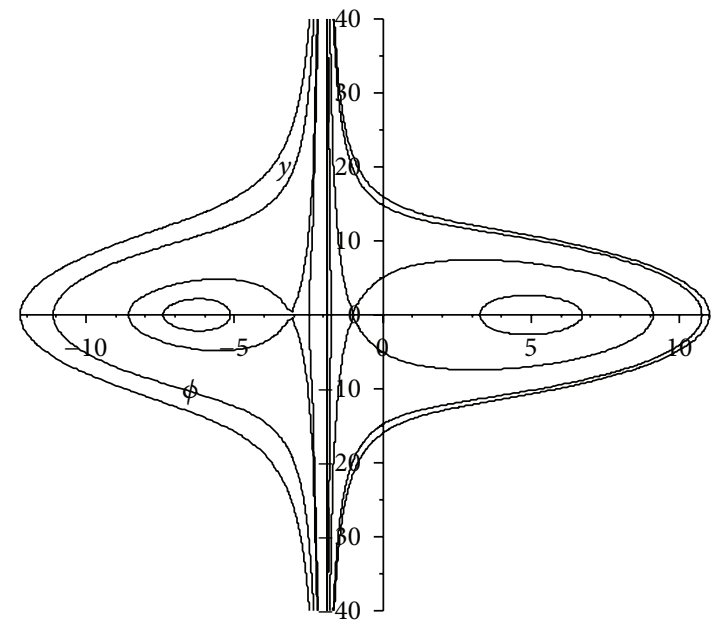

(b)

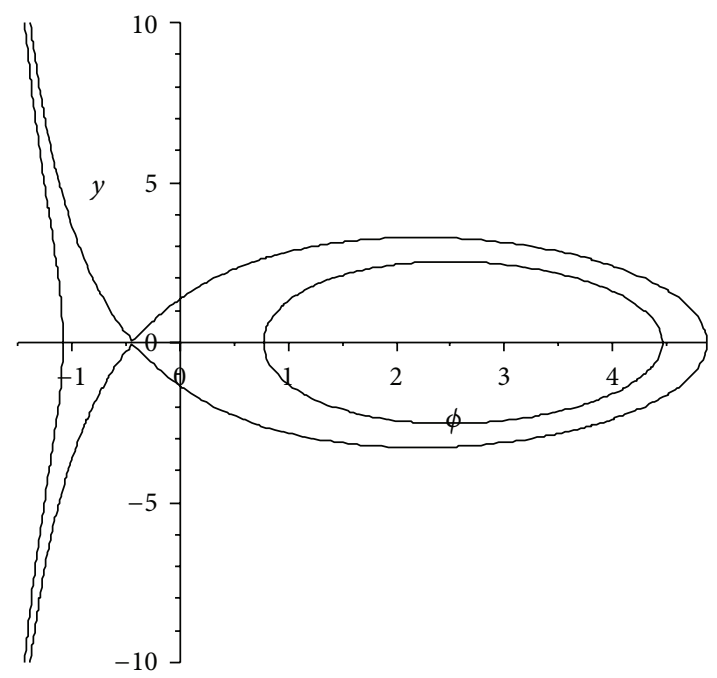

(c)

Figure 1: Phase portraits of (10) for $\phi \neq-c$. (a) The case for $-(1 / 2) c^{2} \leq g<(1 / 4) c^{2}, F\left(\phi_{+}\right)<0$. (b) The case for $g<-(1 / 2) c^{2}, F\left(\phi_{-}\right)<$ $0, F\left(\phi_{+}\right)<0$. (c) The case for $g<-(1 / 2) c^{2}, F\left(\phi_{-}\right)>0, F\left(\phi_{+}\right)<0$.

By using the results of the above lemmas and the basic theory of the singular nonlinear traveling wave equations [22], we obtain the dynamical behavior of the traveling wave solutions of (1) as follows.

\subsection{Solitary Waves Solutions to (1)}

Proposition 5. There exists a smooth bell-shape solitary wave solution of the first component of (1) if one takes plus sign in (1) and one of the following conditions is satisfied:

$$
\begin{aligned}
& \text { (1) }-(1 / 2) c^{2} \leq g<(1 / 4) c^{2}, F\left(\phi_{+}\right)<0 ; \\
& \text { (2) } g<-(1 / 2) c^{2}, F\left(\phi_{-}\right)<0, F\left(\phi_{+}\right)<0 \text {; } \\
& \text { (3) } g<-(1 / 2) c^{2}, F\left(\phi_{-}\right)>0, F\left(\phi_{+}\right)<0 .
\end{aligned}
$$

Those solitary wave solutions are corresponding to the homoclinic orbit given by $H(\phi, y)=h_{1}$ to the saddle point
$E_{1}$ in Figures 1(a) and 1(c) and by $H(\phi, y)=h_{3}$ to the saddle point $E_{3}$ in Figure 1(b). For simplicity, we only give one planar profile of the first component $u$ in Figure 3(a). The second component of (1) is then given according to (8).

Proposition 6. There exists a smooth valley-shape solitary wave solution of the first component of (1) and one of the following conditions is satisfied:

(1) $g<-(1 / 2) c^{2}, F\left(\phi_{-}\right)<0, F\left(\phi_{+}\right)<0$ and taking plus sign in (1);

(2) $F(c)>0,-(1 / 2) c^{2} \leq g<(1 / 4) c^{2}, F\left(\phi_{+}\right)<0$ and taking minus sign in (1);

(3) $F(c)<0, g<-(1 / 2) c^{2}, F\left(\phi_{-}\right)<0, F\left(\phi_{+}\right)<0$ and taking minus sign in (1). 


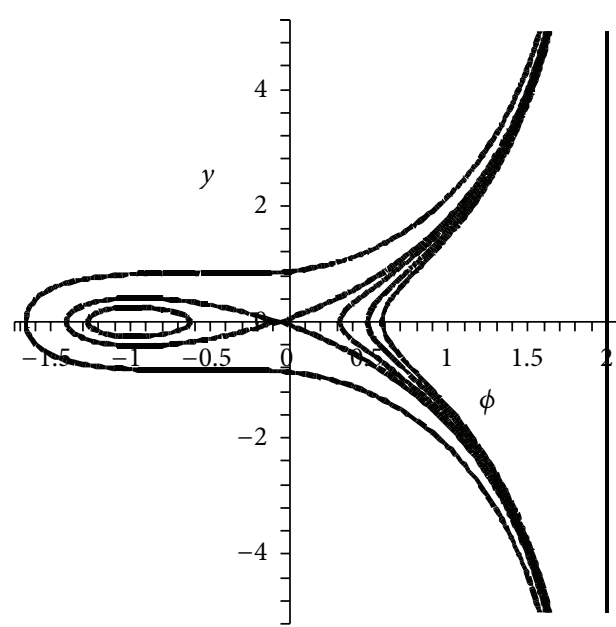

(a)

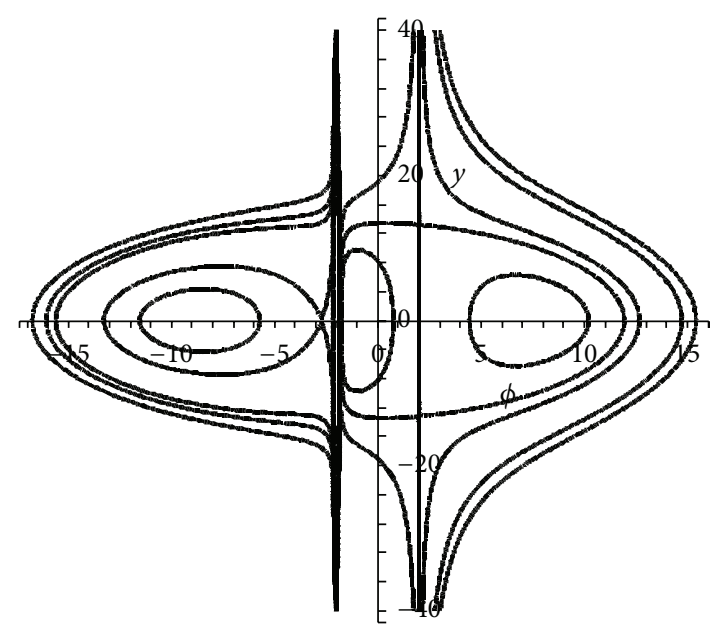

(b)

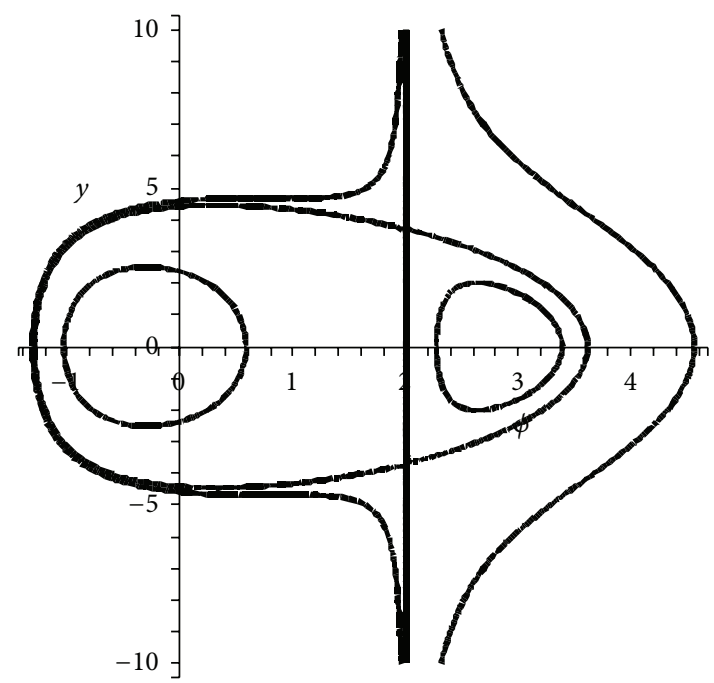

(c)

Figure 2: Phase portraits of (10) for $\phi \neq-c$, taking minus sign. (a) The case for $F(c)>0,-(1 / 2) c^{2} \leq g<(1 / 4) c^{2}, F\left(\phi_{+}\right)<0$. (b) The case for $F(c)<0, g<-(1 / 2) c^{2}, F\left(\phi_{-}\right)<0, F\left(\phi_{+}\right)<0$. (c) The case for $F(c)<0, g<-(1 / 2) c^{2}, F\left(\phi_{-}\right)>0, F\left(\phi_{+}\right)<0$.

These smooth valley-shape solitary wave solutions are corresponding to the homoclinic orbit given by $H(\phi, y)=h_{2}$ to the saddle point $E_{2}$ in Figures 3(a), 2(a), and 2(b). A planar profile of the first component $u$ is shown in Figure 4(a).

\subsection{Smooth Periodic Solutions}

Proposition 7. There is a family of smooth periodic wave solutions of (1) if one of the following conditions is satisfied:

(1) $-(1 / 2) c^{2} \leq g<(1 / 4) c^{2}, F\left(\phi_{+}\right)<0$ and taking plus sign in (1);

(2) $g<-(1 / 2) c^{2}, F\left(\phi_{-}\right)<0, F\left(\phi_{+}\right)<0$ and taking plus sign in (1);

(3) $g<-(1 / 2) c^{2}, F\left(\phi_{-}\right)<0, F\left(\phi_{+}\right)<0$ and taking plus sign in (1);
(4) $g<-(1 / 2) c^{2}, F\left(\phi_{-}\right)>0, F\left(\phi_{+}\right)<0$ and taking plus sign in (1);

(5) $F(c)>0,-(1 / 2) c^{2} \leq g<(1 / 4) c^{2}, F\left(\phi_{+}\right)<0$ and taking minus sign in (1);

(6) $F(c)<0, g<-(1 / 2) c^{2}, F\left(\phi_{-}\right)<0, F\left(\phi_{+}\right)<0$ and taking minus sign in (1);

(7) $F(c)<0, g<-(1 / 2) c^{2}, F\left(\phi_{-}\right)<0, F\left(\phi_{+}\right)<0$ and taking minus sign in (1);

(8) $F(c)<0, g<-(1 / 2) c^{2}, F\left(\phi_{-}\right)<0, F\left(\phi_{+}\right)<0$ and taking minus sign in (1);

(9) $F(c)<0, g<-(1 / 2) c^{2}, F\left(\phi_{-}\right)>0, F\left(\phi_{+}\right)<0$ and taking minus sign in (1);

(10) $F(c)<0, g<-(1 / 2) c^{2}, F\left(\phi_{-}\right)>0, F\left(\phi_{+}\right)<0$ and taking minus sign in (1). 


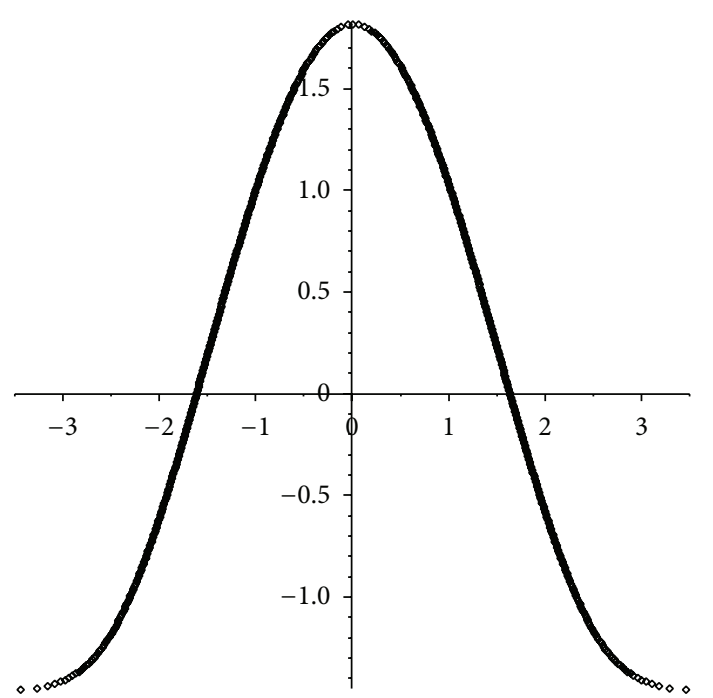

(a) Planar profiles of solutions to $u$

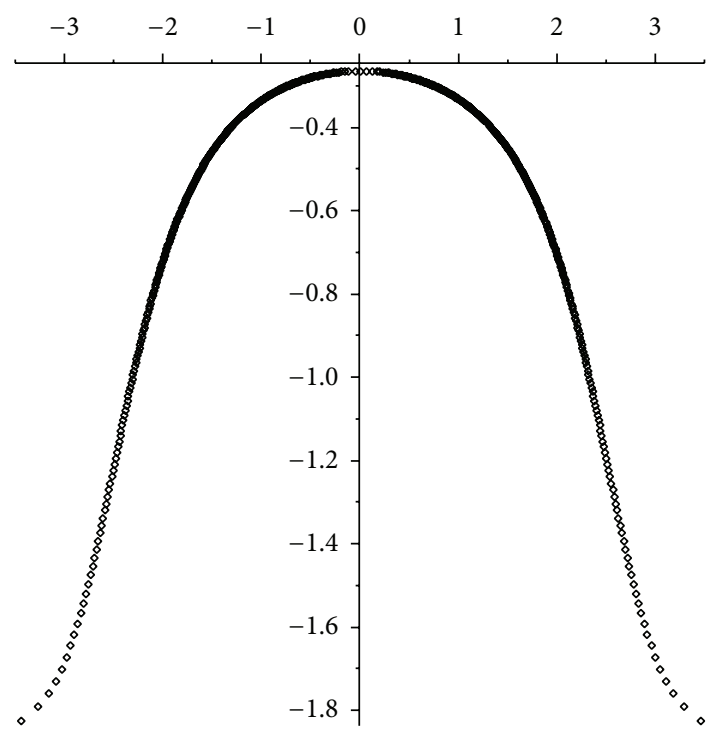

(b) Planar profiles of solutions to $v$

FIGURE 3: Smooth bell-shape solitary wave solution.

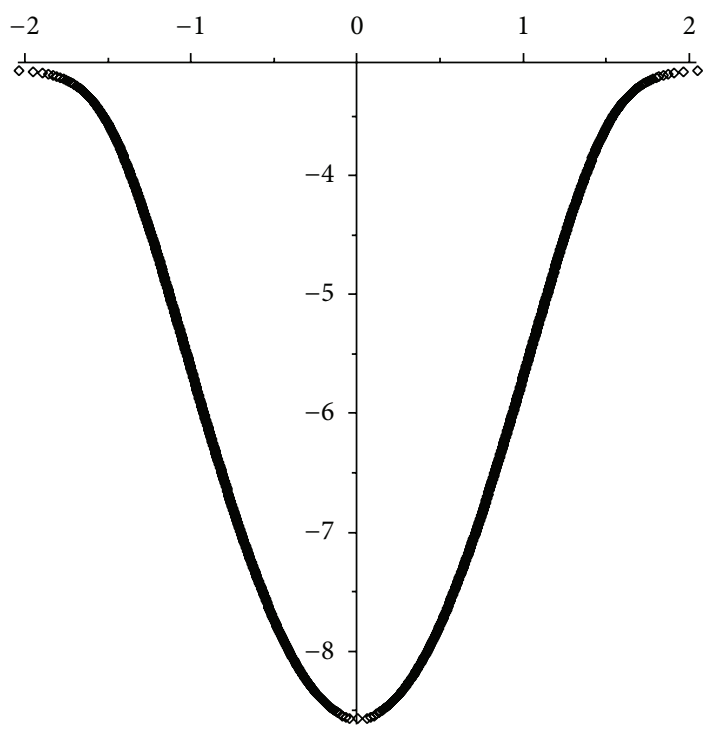

(a) Planar profiles of solutions to $u$

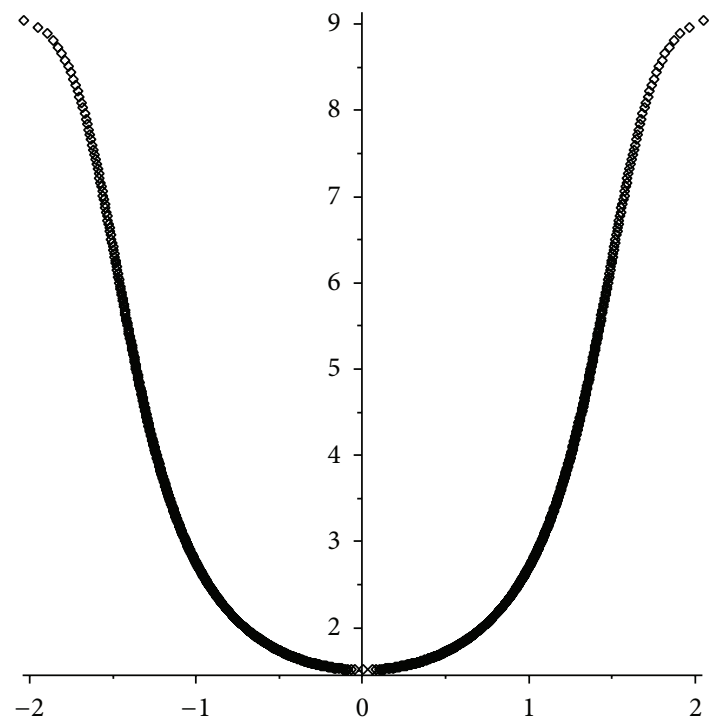

(b) Planar profiles of solutions to $v$

FIGURE 4: Smooth valley-shape solitary wave solution.

Those periodic traveling wave solutions correspond to the family of smooth periodic orbits surrounding the centers in Figures 1 and 2. A planar profile of the first component $u$ is shown in Figure 5(a).

3.3. Nonsmooth Periodic Wave Solution. In Figures 1(b) and $1(\mathrm{c})$, the singular straight line $\phi=c$ intersects with the close orbit. By theory of the singular nonlinear traveling wave equations [22], there are nonsmooth wave solutions to (1).
Proposition 8. There is a peaked periodic cusp wave solution if one takes minus sign in (1) and one of the following conditions is satisfied:

$$
\begin{aligned}
& \text { (1) } F(c)<0, g<-(1 / 2) c^{2}, F\left(\phi_{-}\right)<0, F\left(\phi_{+}\right)<0 \text {; } \\
& \text { (2) } F(c)<0, g<-(1 / 2) c^{2}, F\left(\phi_{-}\right)>0, F\left(\phi_{+}\right)<0 .
\end{aligned}
$$

These peaked periodic cusp wave solutions are corresponding to the arch curve in the left side of $\phi=c$ passing through 


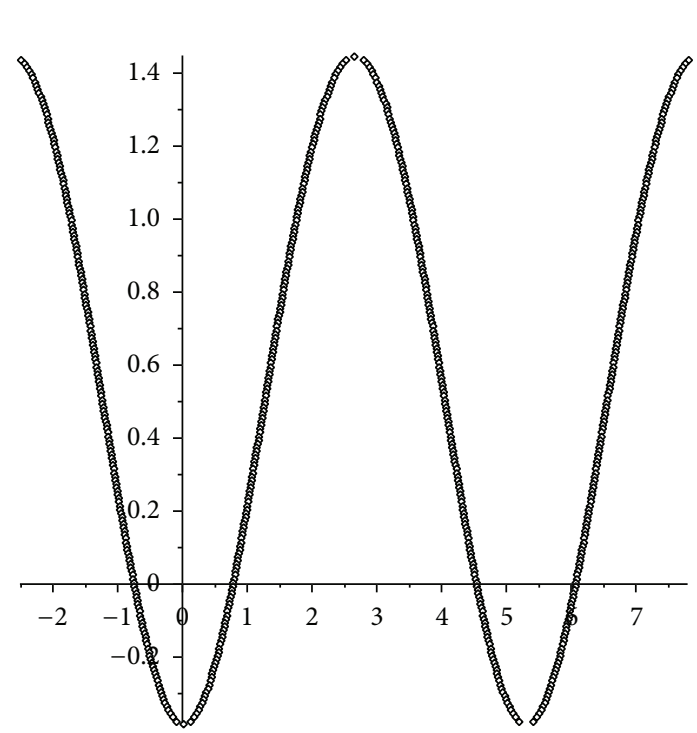

(a) Planar profiles of solutions to $u$

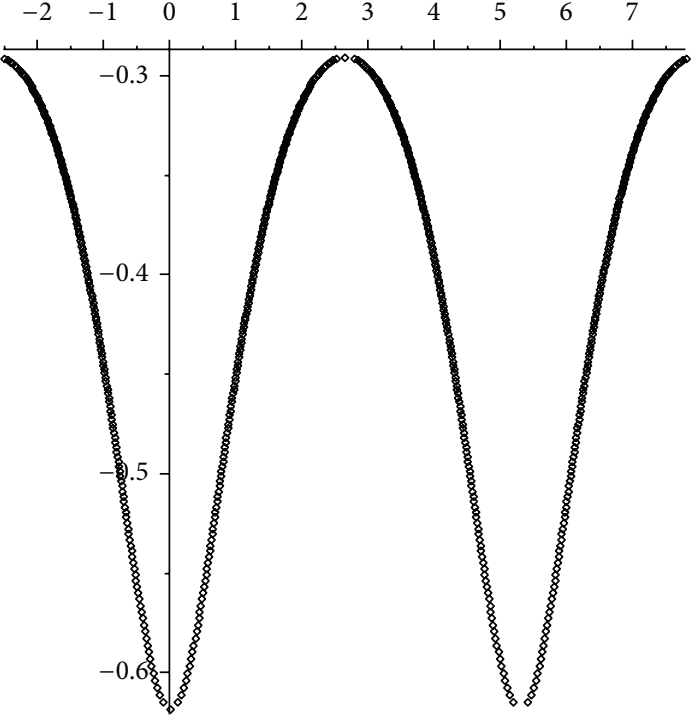

(b) Planar profiles of solutions to $v$

FIGURE 5: Smooth periodic wave solutions.

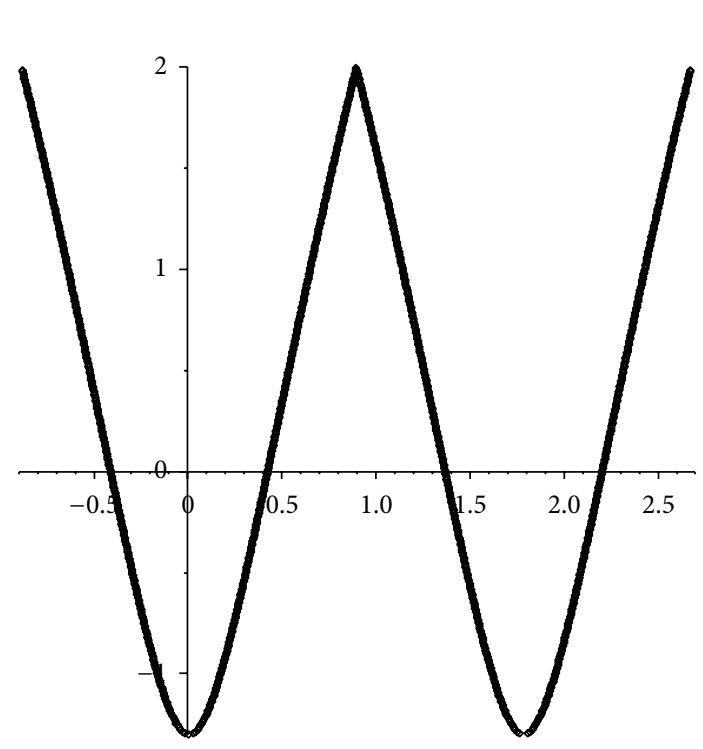

(a) Planar profiles of solutions to $u$

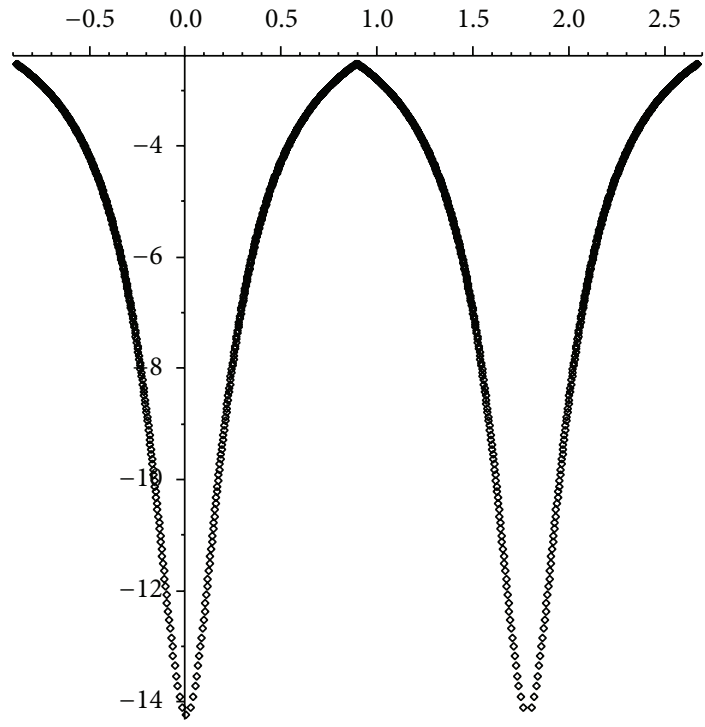

(b) Planar profiles of solutions to $v$

FIGURE 6: Peaked periodic cusp wave solution.

the singular saddle points $E_{5}$ and $E_{6}$ and surrounding the center $E_{3}$ in Figure 2(b) and that passing through the singular saddle points $E_{3}$ and $E_{4}$ and surrounding the center $E_{1}$ in Figure 2(c), respectively. Profiles are shown in Figure 6(a).

Proposition 9. There is a valley-shape periodic cusp wave solution if one takes minus sign in (1) and one of the following conditions is satisfied.

(1) $F(c)<0, g<-(1 / 2) c^{2}, F\left(\phi_{-}\right)<0, F\left(\phi_{+}\right)<0$;

(2) $F(c)<0, g<-(1 / 2) c^{2}, F\left(\phi_{-}\right)>0, F\left(\phi_{+}\right)<0$.
Those valley-shape periodic cusp wave solutions are corresponding to the arch curve in the right side of $\phi=c$ passing through the singular saddle points $E_{5}$ and $E_{6}$ and surrounding the center $E_{4}$ in Figure 2(b) and that passing through the singular saddle points $E_{3}$ and $E_{4}$ and surrounding the center $E_{2}$ in Figure 2(c). Profiles are shown in Figure 7(a).

\section{Conclusions}

By using theory of the singular nonlinear traveling wave equations, we found the existence of several different kinds of traveling wave solutions of (1). It is shown that the signs 


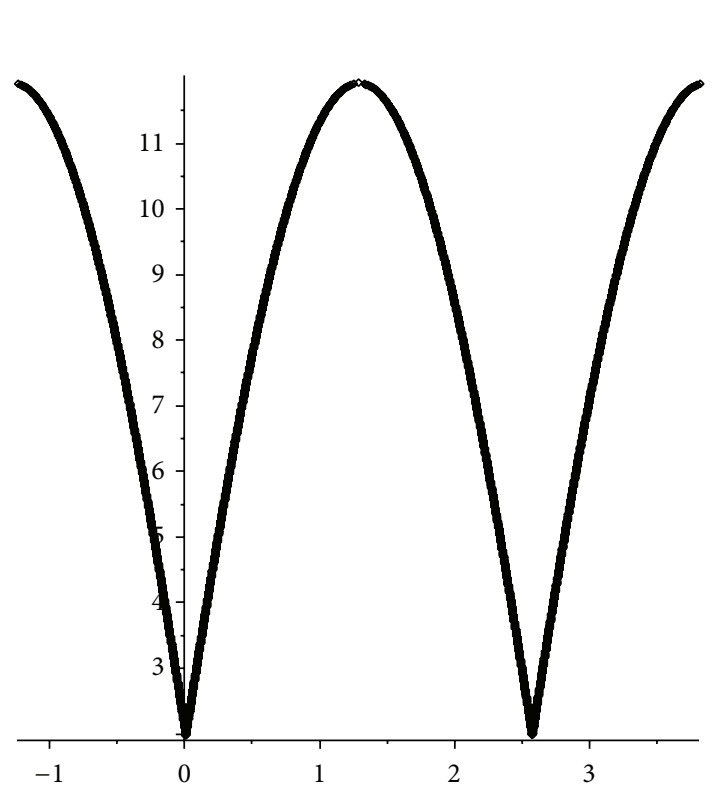

(a) Planar profiles of solutions to $u$

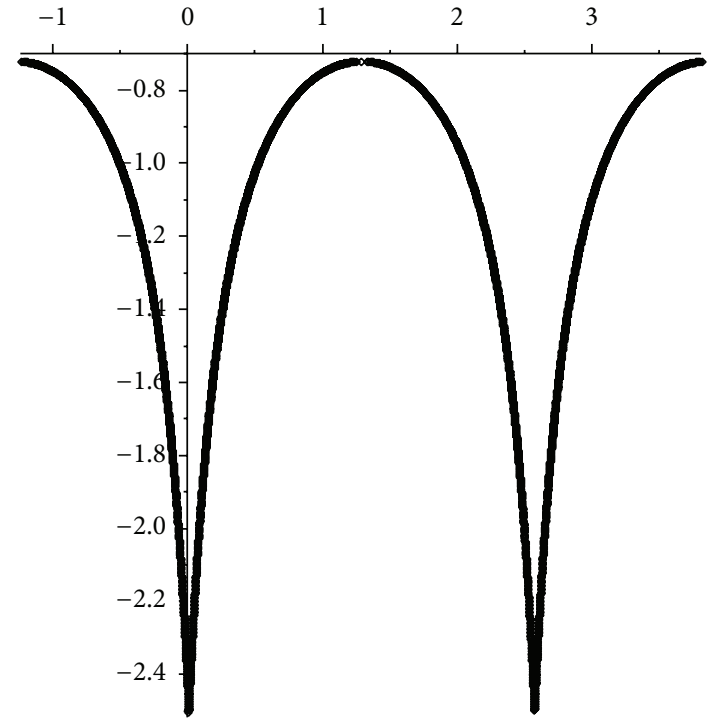

(b) Planar profiles of solutions to $v$

FIGURE 7: Valley-shape periodic cusp wave solution.

had some influence on the type of solution. There are only smooth traveling wave solutions when taking plus sign. Nonsmooth traveling wave solutions arise when the sign changes to minus. Furthermore, no peakons have been found in our work although the two-component dual Ito equation is analogous to the two-component Camassa-Holm equation.

\section{Conflict of Interests}

The authors declare that there is no conflict of interests regarding the publication of this paper.

\section{Acknowledgment}

Research was supported by the National Natural Science Foundation of China (no. 11026169).

\section{References}

[1] C. Guan and Z. Yin, "Global existence and blow-up phenomena for an integrable two-component Camassa-Holm shallow water system," Journal of Differential Equations, vol. 248, no. 8, pp. 2003-2014, 2010.

[2] G. Gui and Y. Liu, "On the global existence and wave-breaking criteria for the two-component Camassa-Holm system," Journal of Functional Analysis, vol. 258, no. 12, pp. 4251-4278, 2010.

[3] O. G. Mustafa, "On smooth traveling waves of an integrable two-component Camassa-Holm shallow water system," Wave Motion, vol. 46, no. 6, pp. 397-402, 2009.

[4] A. Constantin and R. I. Ivanov, "On an integrable twocomponent Camassa-Holm shallow water system," Physics Letters A, vol. 372, no. 48, pp. 7129-7132, 2008.

[5] J. B. Li and Y. S. Li, "Bifurcations of travelling wave solutions for a two-component Camassa-Holm equation," Acta Mathematica Sinica, vol. 24, no. 8, pp. 1319-1330, 2008.
[6] X. Fan, S. Yang, J. Yin, and L. Tian, "Bifurcations of traveling wave solutions for a two-component Fornberg-Whitham equation," Communications in Nonlinear Science and Numerical Simulation, vol. 16, no. 10, pp. 3956-3963, 2011.

[7] P. Guha and P. J. Olver, "Geodesic flow and two (super) component analog of the Camassa-Holm equation," Symmetry Integrability and Geometry Methods and Applications, vol. 2, article 054, 2006.

[8] M. Ito, "Symmetries and conservation laws of a coupled nonlinear wave equation," Physics Letters A, vol. 91, no. 7, pp. 335-338, 1982.

[9] R. Camassa, D. D. Holm, and J. M. Hyman, "A new integrable shallow water equation," Advances in Applied Mechanics, vol. 31, pp. 1-33, 1994.

[10] R. Camassa and D. D. Holm, "An integrable shallow water equation with peaked solitons," Physical Review Letters, vol. 71, no. 11, pp. 1661-1664, 1993.

[11] C. A. Gomez S, "New traveling waves solutions to generalized Kaup-KUPershmidt and Ito equations," Applied Mathematics and Computation, vol. 216, no. 1, pp. 241-250, 2010.

[12] F. Khani, "Analytic study on the higher order Ito equations: new solitary wave solutions using the Exp-function method," Chaos, Solitons \& Fractals, vol. 41, no. 4, pp. 2128-2134, 2009.

[13] D. Li and J. Zhao, "New exact solutions to the $(2+1)$ dimensional Ito equation: extended homoclinic test technique," Applied Mathematics and Computation, vol. 215, no. 5, pp. 19681974, 2009.

[14] S. F. Tian and H. Q. Zhang, "Riemann theta functions periodic wave solutions and rational characteristics for the $(1+1)$ dimensional and $(2+1)$-dimensional Ito equation," Chaos, Solitons \& Fractals, vol. 47, pp. 27-41, 2013.

[15] Z. Zhao, Z. Dai, and C. Wang, "Extend three-wave method for the $(1+2)$-dimensional Ito equation," Applied Mathematics and Computation, vol. 217, no. 5, pp. 2295-2300, 2010.

[16] Y. Zhang, Y. C. You, W. X. Ma, and H. Q. Zhao, "Resonance of solitons in a coupled higher-order Ito equation," Journal of 
Mathematical Analysis and Applications, vol. 394, no. 1, pp. 121128, 2012.

[17] H. Zhao, "Soliton solution of a multi-component higher-order Ito equation," Applied Mathematics Letters, vol. 26, no. 7, pp. 681686, 2013.

[18] A. Chen, S. Wen, S. Tang, W. Huang, and Z. Qiao, "Effects of quadratic singular curves in integrable 5 equations," To appear in Studies in Applied Mathematics.

[19] L. Perko, Differential Equations and Dynamical Systems, vol. 7 of Texts in Applied Mathematics, Springer, New York, NY, USA, 1991.

[20] G. Betchewe, B. B. Thomas, K. K. Victor, and K. T. Crepin, "Dynamical survey of a generalized-Zakharov equation and its exact travelling wave solutions," Applied Mathematics and Computation, vol. 217, no. 1, pp. 203-211, 2010.

[21] K. Gatermann and S. Hosten, "Computational algebra for bifurcation theory," Journal of Symbolic Computation, vol. 40, no. 4-5, pp. 1180-1207, 2005.

[22] J. B. Li and H. H. Dai, On the Study of Singular Nonlinear Travelling Wave Equations: Dynamical Approach, Science Press, Beijing, China, 2007.

[23] Y. Feng, W. Shan, W. Sun, H. Zhong, and B. Tian, "Bifurcation analysis and solutions of a three-dimensional KudryashovSinelshchikov equation in the bubbly liquid," Communications in Nonlinear Science and Numerical Simulation, vol. 19, no. 4, pp. 880-886, 2014.

[24] H. Liu and J. Li, "Symmetry reductions, dynamical behavior and exact explicit solutions to the Gordon types of equations," Journal of Computational and Applied Mathematics, vol. 257, pp. 144-156, 2014. 


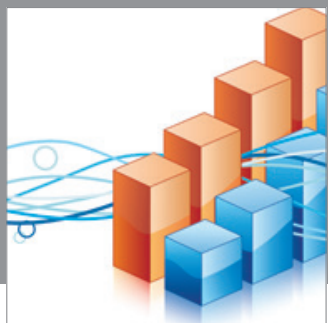

Advances in

Operations Research

mansans

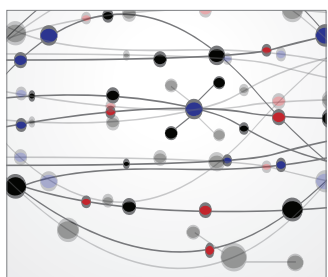

The Scientific World Journal
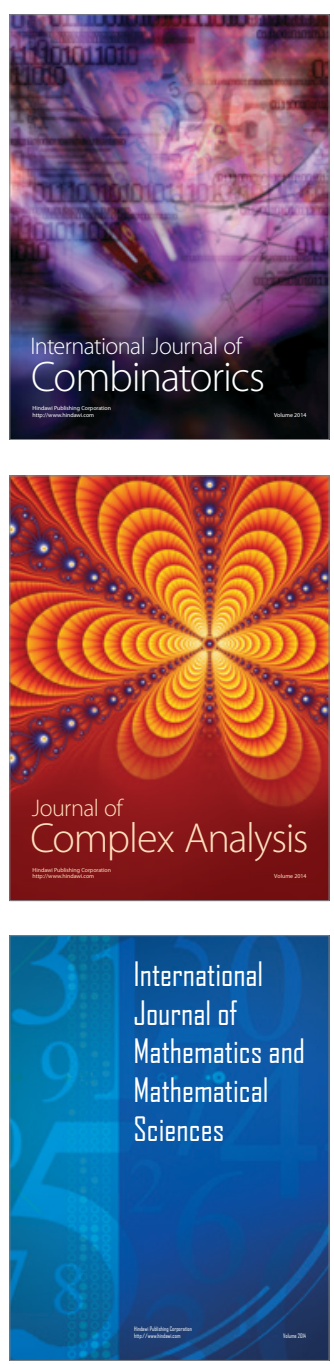
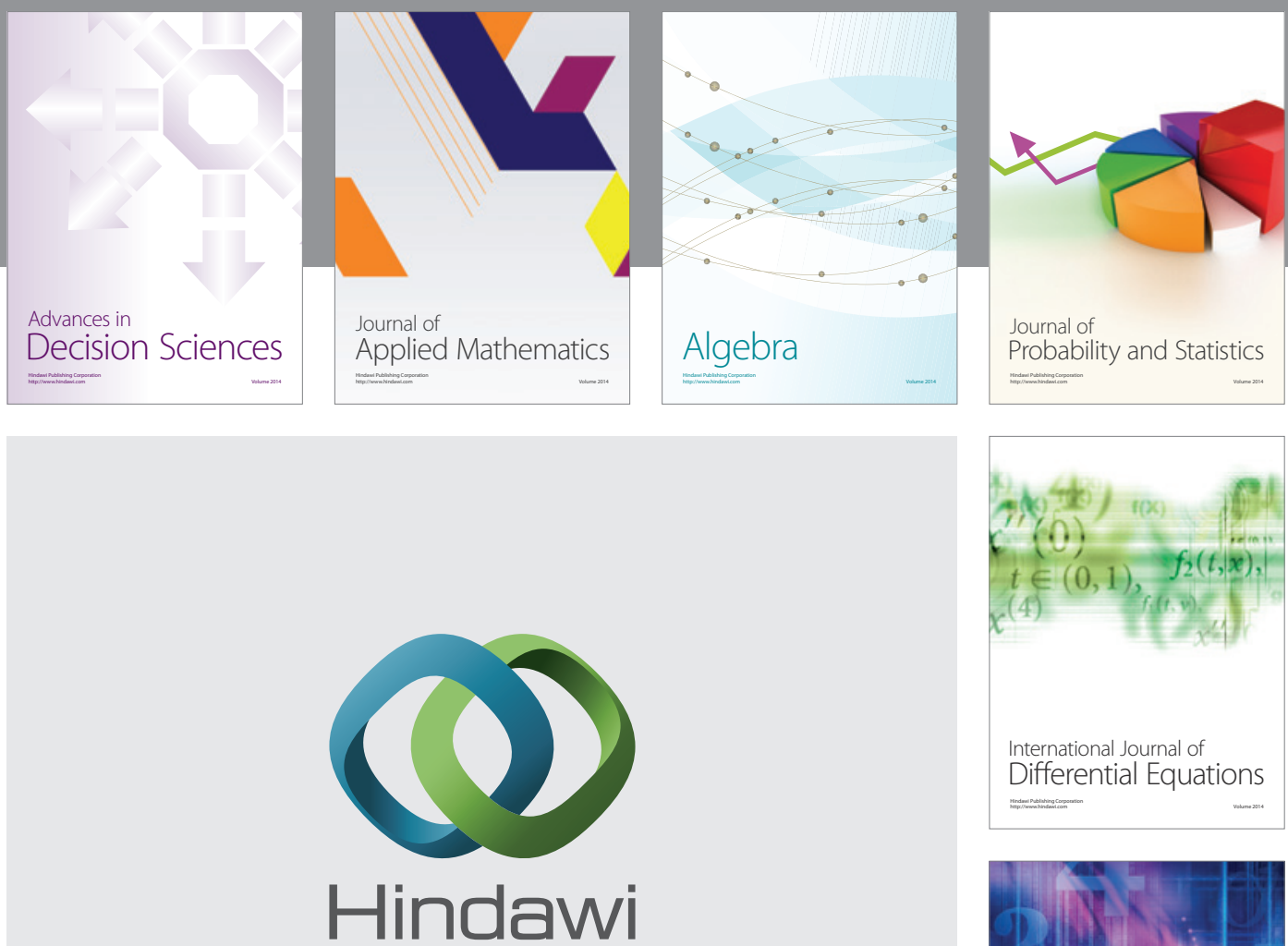

Submit your manuscripts at http://www.hindawi.com
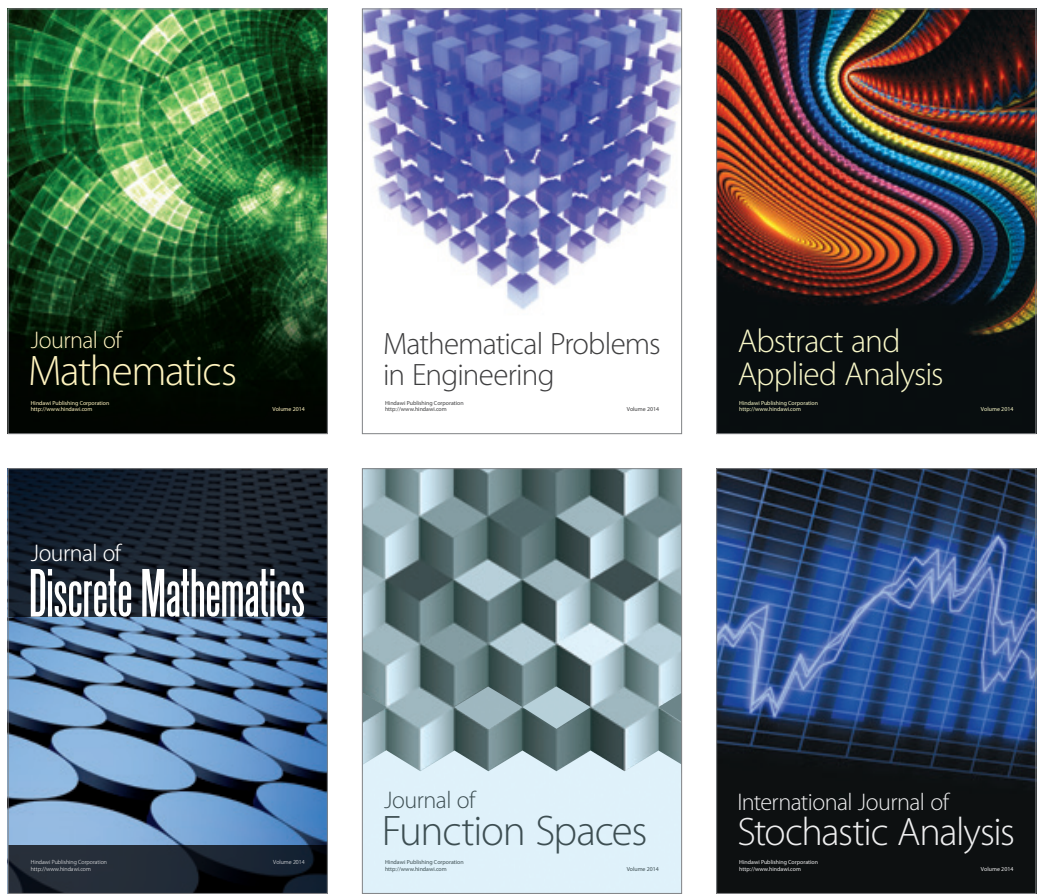

Journal of

Function Spaces

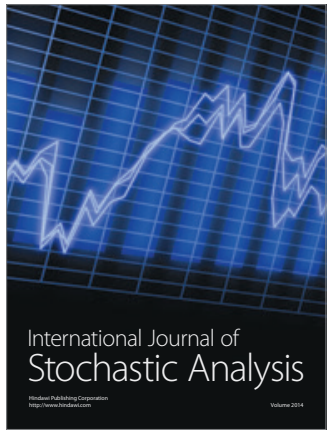

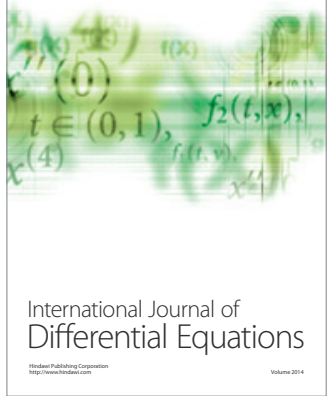
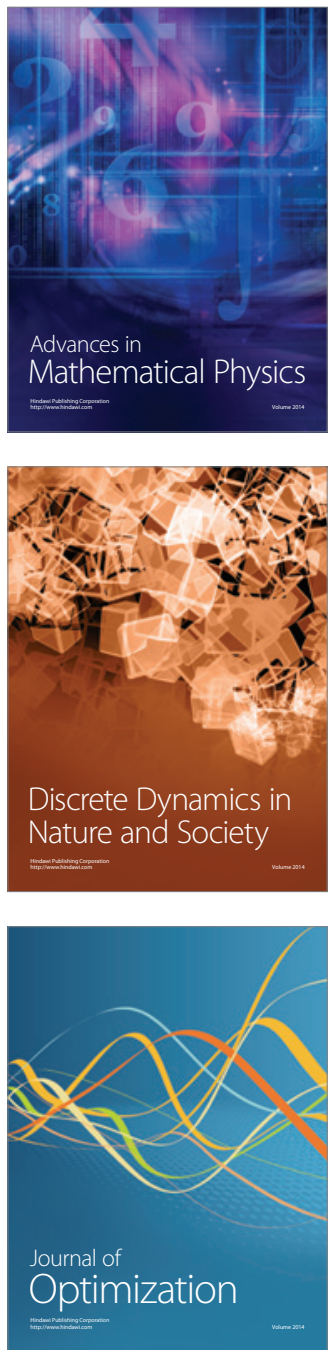